\section{Also Human: The Inner Lives of Doctors}

\author{
Caroline Elton
}

New York, NY: Basic Books, 2018, 336 pages, \$30.00; ISBN-10: 0465093736; ISBN-13: 978-0465093731

Physicians save lives. That is the demanding standard every physician is held to and the one they always strive to meet, despite the uncertainty and fallibility that they face. Patients' lives are constantly put into their hands without question, and physicians bear that responsibility proudly. Yet physicians, too, face their own ordeals. They, too, need saving.

Also Human: The Inner Lives of Doctors is a collection of accounts of the stress and hardships physicians face, told as a striking indictment of the culture surrounding medical practice. It is the culmination of Dr. Caroline Elton's career as a psychologist, organizing the stories and experiences of her clients and herself, combined with published literature, into a narrative that exposes real issues and presents a glimmer of hope for progress. Dr. Elton provided guidance to hundreds of physicians over the course of her extensive career, and even learned from them. In essence, she was the doctor to doctors-doctors who are too often believed to be free from common ills, both physical and mental. Throughout her book, she tackles major issues facing physicians, some many might not have thought existed, one by one, with learned authority and vivid detail.

The first few chapters of the book address the issues plaguing medical school graduates and new physicians. Though Dr. Elton's practice was based primarily in the United Kingdom, she does her due diligence in discussing the reality of the American medical education, occasionally comparing these systems to other countries in an effort to highlight alternatives and solutions. The transition in the United Kingdom and the United States for medical students to hospitals is characterized by uncertainty. An account from one of Dr. Elton's clients, Hilary, reveals the first day working in a hospital that left this new unprepared doctor bearing the sole responsibility of a patient potentially on their death bed and 9 more patients she was too panicked to handle, accompanied by unsympathetic nurses and absent senior physicians. Accounts and statistics reveal that medical students in the United Kingdom who are in the greatest need of support are often sent to hospitals far away and given almost no social support system. Lack of support and sympathy extend into poor working conditions for physicians that lead to burnout, with few workable solutions being implemented.
Another interesting account reveals an inexperienced and untrained physician, Vartika, being given the responsibility of certifying the death of an infant while being the least qualified member of the medical team to do so. As in the case, Dr. Elton insists that physicians are not immune to psychologic trauma and vulnerability affecting their work, especially as it relates to patients and other physicians. Recognizing warning signs and extending empathy to other physicians, especially junior physicians, can be dangerously lacking in hospital environments.

The issue of sexuality as a physician is one seldom discussed. For one of Dr. Elton's clients who was a gay obstetrician, that was out of the question. For medical students, the reality of sexual responses, especially for those who are lacking experience with the human body, is ignored. Dr. Elton explores the denial of medical education and training in addressing physicians as sexual people. Sexual inexperience or repression in doctors can be dangerous to both them and their patients, and at least in the United Kingdom, guidelines do not address the issue.

Throughout the rest of the book, Dr. Elton builds a convincing argument against the social culture of the medical community, between an obstetrician experiencing censure for expressing distress while trying to have a child, a medical director with cerebral palsy experiencing stereotyping, and of course, rampant sexism and racism. She ends the book with chapters on the difficulty of leaving and entering the profession, which are less poignant and compelling than the other chapters, but nonetheless relevant.

As Caroline Elton demonstrates in her book, there is a wealth of literature pointing to the very issues she outlines, but she provides human stories to every issue, making her case pertinent and compelling. Her clients are unfortunately not alone in their experiences. Also Human: The Inner Lives of Doctors reminds every physician and patient that doctors are not superhuman.

Vikrant R. Tadepalli

Johns Hopkins University

Baltimore, Maryland

Robert Matthews*

*Stony Brook University Hospital

HSC Building Level 4, Room 120

Stony Brook, NY 11794

E-mail: Robert.matthews@stonybrook.edu

Published online Jun. 14, 2019.

DOI: 10.2967/jnumed.119.232371 